\title{
Die Bedeutung von Natriumhydrogenkarbonat
}

Der Säure-Basen-Haushalt und Körperfunktionen sind eng verknüpft. Eine metabolische Azidose ( $\mathrm{mA}$ ) hat viele negative Auswirkungen, auch auf die Nierenfunktion. Die $\mathrm{mA}$ ist in ihren unterschiedlichen Schweregraden mittels Blutgasanalyse leicht zu enttarnen. Ein Standardbikarbonat von unter $22 \mathrm{mmol} / \mathrm{l}$ gilt als kritisch und sollte Anlass sein, die Ursachen zu suchen, diese zu behandeln und gegen die Azidose vorzugehen. Am besten eignen sich orale, magensaftresistente Natriumhydrogenkarbonat-Präparate.

\section{Natriumhydrogenkarbonat}

\section{Die Chemie}

Natriumhydrogenkarbonat (umgangssprachlich Natron) ist das Natriumsalz der Kohlensäure mit der Summenformel $\mathrm{NaHCO}_{3}$. Dafür finden auch die Begriffe $\mathrm{Na}$ triumbikarbonat oder Bikarbonat Verwendung. Natriumkarbonat steht für $\mathrm{Na}_{2} \mathrm{CO}_{3}$ (umgangssprachlich Soda); im Handel u.a. angeboten unter Backsoda, Speisesoda, Speisenatron oder anderen Markennamen. Diverse Bezeichnungen erschweren es, Natron $\left(\mathrm{NaHCO}_{3}\right)$ und Soda $\left(\mathrm{Na}_{2} \mathrm{CO}_{3}\right)$ auseinander zu halten. Bikarbonat aber ist chemisch das einfach deprotonierte Salz $\left(\mathrm{H}^{+} \hat{=}\right.$ Proton) der Kohlensäure $\left(\mathrm{H}_{2} \mathrm{CO}_{3}\right)$ mit der Formel $\mathrm{HCO}_{3}{ }^{-}$.

\section{Viele Einsatzmöglichkeiten}

$\mathrm{NaHCO}_{3}$ ist Bestandteil in Feinwasch- und Putzmitteln, Back- und Brausepulver und im Feuerlöschpulver, ist Hilfsmittel bei der Käsereifung, dient zur Schaffung einer brandhemmenden Atmosphäre, z. B. in Flugschreibern, und als Lebensmittelzusatzstoff E500. $\mathrm{NaHCO}_{3}$ wird angewendet z. B. als Bullrich-Salz ${ }^{\circledR}$ oder Kaiser Natron ${ }^{\circledR}$ gegen Sodbrennen. Mit Säuren (z. B. Magensäure) reagiert Natriumhydrogenkarbonat (also Natron) zu Natriumchlorid ( $\mathrm{NaCl}$ : Kochsalz), Kohlenstoffdioxid $\left(\mathrm{CO}_{2}\right)$ und Wasser $\left(\mathrm{H}_{2} \mathrm{O}\right)$.

\section{REAKTIONSGLEICHUNG}

$\mathrm{NaHCO}_{3}+\mathrm{HCl} \rightarrow \mathrm{NaCl}+\mathrm{CO}_{2}+\mathrm{H}_{2} \mathrm{O}$
Die Möglichkeit, Säureüberschüsse durch Bikarbonat $\left(\mathrm{HCO}_{3}{ }^{-}\right)$zu puffern, ist für den menschlichen Organismus überlebenswichtig [1].

\section{Bikarbonat}

\section{Die Rolle im menschlichen Organismus}

Die physiologische Wasserstoff-Ionen-Konzentration halten 3 Regulationsvorgänge in dem engen $\mathrm{pH}$-Wert-Bereich von 7,377,45 konstant aufrecht ( $\vee$ Tab. 1 ). Dazu gehören:

- die Pufferung durch Puffersubstanzen

- die Pufferung durch die respiratorische Regulation beim Abatmen von $\mathrm{CO}_{2}$

- die renale Pufferung durch die Ausscheidung saurer Wasserstoffionen

Die Pufferreaktionen selbst erfolgen:

- intrazellulär durch Phosphat $\left(\mathrm{HPO}_{4}{ }^{2-}\right)$ und Hämoglobin

- extrazellulär durch Bikarbonat $\left(\mathrm{HCO}_{3}{ }^{-}\right)$ und Plasmaproteine

Das von der Lungen- und Nierenfunktion regulierte Kohlendioxid/Bikarbonat-Puffersystem $\left(\mathrm{CO}_{2} / \mathrm{HCO}_{3}{ }^{-}\right)$macht $75 \%$ der Gesamtpufferkapazität aus, hat damit die wichtigste Stellung im Säure-Basen-Haushalt inne [1]. Im Säure-Basen-Haushalt gibt es 3 Gruppen von Störungen:

- metabolische Störungen

- respiratorische Störungen

- gemischte Störungen (metabolische und respiratorische Störungen)

\section{Das „Standardbikarbonat“: wichti- ger Parameter der Blutgasanalyse}

Man unterscheidet die Alkalose und die Azidose. Die akute und die chronische metabolische Azidose ( $\mathrm{cmA}$ ) entsteht durch die stoffwechselbedingte Übersäuerung des Blutes. Das Risiko dafür haben besonders:

- Patienten mit Nierenfunktionseinschränkung: Die Säure-AusscheidungsKapazität ist reduziert. Patienten im Vordialysestadium und im Dialysestadium entwickeln daher häufig eine chronische metabolische Azidose.
- Tab. 1 Normalbereiche der Blutgasanalyse (BGA).

\begin{tabular}{l|l}
\hline Variable & Wert \\
\hline $\mathrm{pH}-$ Wert & $7,37-7,45$ \\
\hline $\mathrm{PCO}_{2}$ (arteriell) & $32-46 \mathrm{mmHg}$ \\
\hline $\begin{array}{l}\mathrm{pCO}_{2} \text { (gemischt- } \\
\text { venös) }\end{array}$ & $37-50 \mathrm{mmHg}$ \\
\hline $\begin{array}{l}\mathrm{HCO}_{3} \text {-Standard } \\
\text { Bikarbonat (arteriell) }\end{array}$ & $22-26 \mathrm{mmol} / \mathrm{l}$ \\
\hline $\begin{array}{l}\text { Basenabweichung } \\
\text { (BE: Base Excess) }\end{array}$ & $-2,0$ bis $+2,0 \mathrm{mmol} / \mathrm{l}$ \\
\hline
\end{tabular}

- Bei gleichzeitig verminderter Lungenfunktion eskaliert dieses Problem, denn die kompensatorische $\mathrm{CO}_{2}$-Abatmung ist zusätzlich erschwert.

- Patienten mit Diabetes mellitus: Bei Insulinmangel entstehen durch die Energiegewinnung aus Fett (Fettsäuren) reichlich Ketonkörper (Ketose), die zur Ketoazidose führen. Der Diabetes mellitus bedingt zudem ein erhöhtes Risiko für Nierenfunktionsstörungen und deren Folgen bis hin zur Dialysepflicht!

- Alkoholkranke: Ständiger Alkoholabbau führt, besonders bei vermindertem extrazellulärem Volumen, zu einer erhöhten Ketonsäurebildung. Der Ketonsäureüberschuss verursacht eine alkoholische Ketoazidose.

- Extremsportler unter hypoxämischen Bedingungen und Langzeitfastende entwickeln - oft unerwartet - eine gefährliche, meist aktute, Laktatazidose. Auch bei kontraindizierter Biguanideinnahme (glomeruläre Filtrationsrate $(G F R)<30 \mathrm{ml} / \mathrm{min} / 1,73 \mathrm{~m}^{2}$ Körperoberfläche) ist das möglich. Metformin ist dann kontraindiziert.

Vergiftungen mit Azetylsalizylsäure (ab $12 \mathrm{~g})$, Glykol (Kühlerfrostschutzmittel) und Methanol führen zu einer akuten Laktatazidose.

\section{Ein Standardbikarbonat von unter $22 \mathrm{mmol} / \mathrm{l}$ in der Blutgasanalyse (BGA) ist kritisch}

Ein Standardbikarbonat (Ausdruck der BGA: $\mathrm{HCO}_{3}{ }^{-}$-std) von unter $22 \mathrm{mmol} / \mathrm{l}$ signalisiert 
eine azidotische Stoffwechsellage. Die Azidose ist bei einem Blut-pH-Wert von über 7,37 noch kompensiert, darunter dekompensiert ( $\triangleright$ Tab. 2). Ein pH von 7,37-7,1 besteht bei einer schweren (dekompensierten), ein $\mathrm{pH}$ von unter 7,1 bei einer lebensbedrohlichen Azidose.

Außer bei der schweren dekompensierten und lebensbedrohlichen Azidose mit der typischen Kußmaulschen Atmung zeigen die latente und die chronische, unvollständig kompensierte Azidose kaum spezifische Symptome. Wichtig bleibt die Entdeckung dieser Störung. Bei Verdacht ist eine Blutgasanalyse angezeigt, am besten aus arteriellem Blut. Für die Praxis genügt Blut aus dem hyperämisierten Ohrläppchen. Venöses Blut eignet sich auch bei Beachtung der etwas anderen Normwerte.

\section{Folgen der metabolischen Azidose}

Die metabolische Azidose $(\mathrm{mA})$ hat messbare und symptomatische Folgen.

\section{Bereich der Zellen}

- Abnahme von Enzymaktivitäten

- Verquellen der Zellen, Verformung von Zellen und Gewebe

- Diffusionsstörungen, dadurch Ernährungsstörung im Gewebe

- Verschlechterung der Sauerstoff-Ausnutzung und damit der Organfunktion

- Anteil bei degenerativen Prozessen

Aminosäuren- und Eiweißstoffwechsel

- erhöhter Eiweißabbau

- Hemmung der Albuminsynthese in der Leber [2]

- Störung der Lymphozytenproliferation (Infektabwehr)

- Reduktion der ATP-vermittelten Auflösung von Tumorzellen

- Aktivitätsverlust der NK-Zellen (natürliche Killerzellen)

\section{Knochenstoffwechsel}

- Steigerung der Knochenresorption $[3,4]$

- Freisetzung von Knochenphosphat zur Gewinnung weiterer Puffersubstanz

- Hemmung der Knochenreparatur $[3,4]$

- Tab. 2 Kompensierte und dekompensierte Azidose.

\begin{tabular}{|l|l|l|}
\hline & kompensiert & dekompensiert \\
\hline $\mathbf{p H}-$ Wert & normal & erniedrigt \\
\hline Standardbikarbonat $\left(\mathrm{HCO}_{3}^{-}\right)$ & erniedrigt & erniedrigt \\
\hline
\end{tabular}

\section{Endokrines System}

- Behinderung der Vitamin-D-HormonAktivierung

- Steigerung der körpereigenen Kortikoid-Sekretion

- Effekte im Schilddrüsenhormonstoffwechsel [5]

- Beeinträchtigung der Erythropoetinwirkung

\section{Natriumhydrogenkarbonat}

\section{Therapie ab Standardbikarbonat von unter $22 \mathrm{mmol} / \mathrm{I}$}

Aus $\mathrm{NaHCO}_{3}$ (Natriumhydrogenkarbonat) in magensaftresistenten Tabletten entstehen im Dünndarm $\mathrm{Na}^{+}$(Natrium) und $\mathrm{HCO}_{3}{ }^{-}$(Bikarbonat) zum Ausgleich der Azidose [6]. Verabreicht wird Natriumhydrogenkarbonat, wirksam ist Bikarbonat. Dieses steht nun dem größten Puffersystem zur Verfügung [1, 6]. Nutzen bringt ein früher Therapiebeginn, also bereits bei der latenten und der nicht dekompensierten Azidose. Die Vorteile sind:

- Vermeiden des Abgleitens in eine dekompensierte metabolische Azidose

- Schutz der stoffwechselintensiven Nierenfunktion

- Verzögerung oder Minderung des Knochenabbaus (wichtig bei Altersosteoporose)

- Korrektur des Ernährungszustandes durch verbesserten Eiweißstoffwechsel

- Verbesserung des Sauerstoffaustausches in den Geweben

- Prävention der linksventrikulären Herzhypertrophie

- Besserung der allgemeinen Abwehrlage (reduziertes Krebsrisiko?)

- allgemeine Steigerung der körperlichen Leistungsfähigkeit

\section{Nutzen früher und ausdauernder Substitution}

Bei chronischer Nierenfunktionseinschränkung

Hier kann die rechtzeitige und v. a. langfristige Behandlung der metabolischen Azidose effektiv zur Erhaltung der Nierenfunktion beitragen. Das ergaben Studien, die eine Verbesserung der Nierenfunktion unter Therapie der metabolischen Azidose durch die orale Gabe von Hydrogenkarbonat zeigten ( $\triangleright$ Abb. 1) [7, 8]. Um den Funktionsverlust der Nieren nachhaltig zu verhindern oder zumindest deutlich zu verzögern, ist die Hydrogenkarbonat-Therapie frühzeitig zu beginnen und langfristig fortzusetzen [8]. Ab dem Unterschreiten eines Wertes von $22 \mathrm{mmol} / \mathrm{l}$ Standardbikarbonat $\left(\mathrm{HCO}_{3}{ }^{-}\right.$-std) in der BGA ist die langfristige Kontrolle des Säure-Basen-Haushalts und dessen Korrektur notwendig.

\section{Bei Niereninsuffizienz und Diabetes mellitus}

In Studien wiesen Patienten mit Insulinresistenz nach einjähriger oraler Therapie mit Natriumhydrogenkarbonat eine Reduktion der Insulinresistenz und damit eine bessere glykämische Kontrolle auf [9].

\section{Im Dialysestadium}

Ziel sollte ein anhaltender Ausgleich der $\mathrm{mA}$ sein. Das ermöglichen ausreichend lange Hämodialysesitzungen, Variationen des Bikarbonatspiegels in der Dialysespüllösung und Einnahme von Natriumhydrogenkarbonat zwischen den Hämodialysen. Trotz regelmäßiger Behandlung mit der Bikarbonatdialyse tendiert der Stoffwechsel im dialysefreien Intervall wieder zur Azidose. Eine Studie zeigte einen positiven Effekt des Ausgleichs im Dialyseintervall [10]. Das wirkt auch auf den Kaliumhaushalt günstig, nicht jeder erhöhte Serum-Kalium-Wert ist allein durch Ernährungsfehler verursacht [11]. 


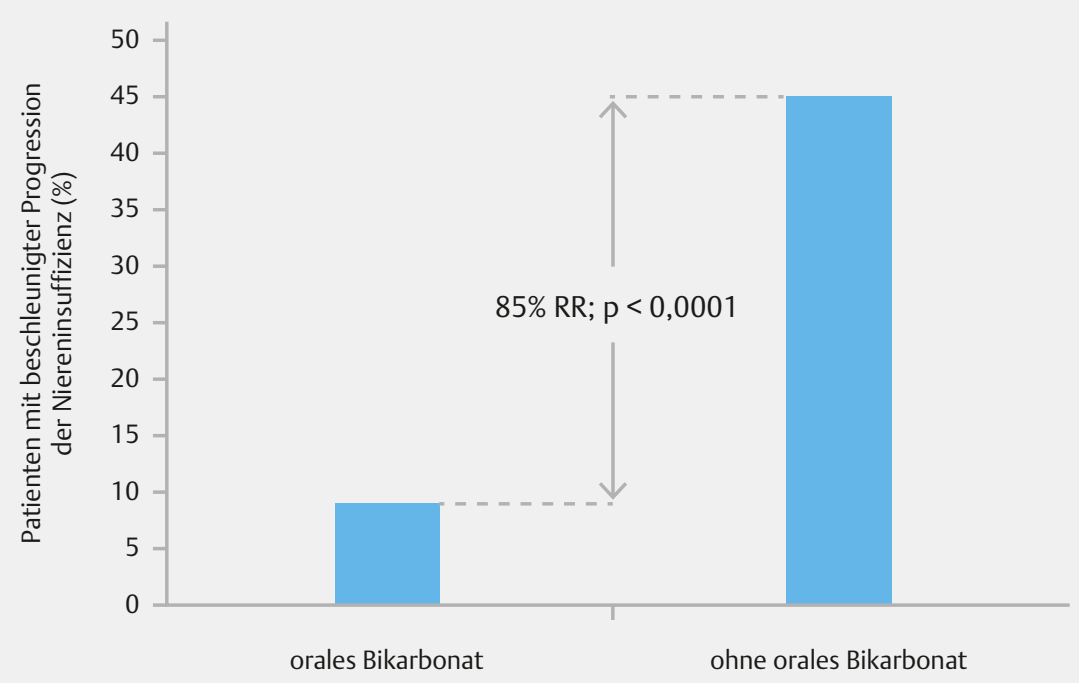

- Abb. 1 Bei oral appliziertem Bikarbonat zeigten sich signifikant weniger Patienten mit beschleunigter Progression der Niereninsuffizienz (modifiziert nach [7]).

\section{Nach Nierentransplantation}

Neue Studien zeigten auch hier eindeutige Vorteile durch eine alkalisierende Ernährung und bei bereits ausgeprägter Azidose durch eine orale Therapie. Die metabolische Azidose erwies sich darin sogar als Risikofaktor für die Langzeitfunktion transplantierter Nieren [12, 13].

\section{Bei Niereninsuffizienz und Schilddrü- senfunktionsstörung}

Eine häufig mit der Nierenfunktionsstörung auftretende endokrine Komplikation ist die Störung der Schilddrüsenfunktion. Natriumhydrogenkarbonat kann offenbar zur Stabilisierung der Schilddrüsenfunktion bei chronischer Nierenkrankheit beitragen [5].

\section{Oral und magensaftresistent}

Neben der Ursachenbeseitigung gewährt der frühe Ausgleich einer cmA den Erhalt der natürlichen Funktions- und Schutzmechanismen im Organismus. Aufmerksamkeit gilt der Ernährung, um das Puffersystem nicht unnötig zu belasten, und andererseits, um die Azidosekorrektur zu unterstützen. Dazu dienen auch Mineralwässer mit einem Hydrogenkarbonatgehalt von mehr als $1000 \mathrm{mg} / \mathrm{l}$.

Für die Substitution von Natriumhydrogenkarbonat eignen sich magensaftresistente Präparate am besten. Die allmähliche Freisetzung im Dünndarm vermeidet den vor- zeitigen Zerfall von $\mathrm{NaHCO}_{3}$ und die damit einhergehende $\mathrm{CO}_{2}$-Bildung im Magen sowie die Beeinträchtigung der bakteriziden Wirkung von Magensäure. Mit einer Tagesdosis von 2000-3000 mg Natriumhydrogenkarbonat ist ein guter Ausgleich des Säure-Basen-Haushalts zu erzielen.

$$
\begin{aligned}
& \text { UMRECHNUNG } \\
& 1,0 \mathrm{~g} \text { Natriumhydrogenkarbo- } \\
& \text { nat }=11,9 \mathrm{mmol} \\
& 1 \mathrm{mmol}=0,084 \mathrm{~g}=84 \mathrm{mg} \mathrm{NaHCO} \\
&
\end{aligned}
$$

Der aktuelle Bedarf lässt sich auch berechnen nach der Formel:

\section{BERECHNUNG}

Bedarf $\mathrm{NaHCO}_{3}$ in $\mathrm{mmol}=$ negativer Basen-Exzess x Körpergewicht $[\mathrm{kg}] / 3$

\section{Fazit}

Für die chronische metabolische Azidose gilt:

- kontrollieren (BGA)

- reagieren

- substituieren (Natriumhydrogenkarbonat)

Dr. med. Dr. Public Health Herbert Stradtmann, Bad Wildungen

\section{Literatur}

[1] Herold G und Mitarbeiter. Säure-BasenHaushalt. In: Herold G und Mitarbeiter. Innere Medizin 2017. 2016: 589-593

[2] Ballmer PE, McNurlan MA, Hulter $\mathrm{HN}$ et al. Chronic metabolic acidosis decreases albumin synthesis and induces negative nitrogen balance in humans. J Clin Invest 1995; 95: 39-45

[3] Lefebvre A, de Vernejoul MC, Gueris J et al. Optimal correction of acidosis changes progression of dialysis osteodystrophy. Kidney Int 1989; 36: 1112-1118

[4] Jehle AM. Metabolische Azidose: Auswirkungen auf den Knochenstoffwechsel. Nieren und Hochdruckkrankheiten 2015; 32: 403-406

[5] Disthabanchong S, Treeruttanawanich A. Oral Sodium Bicarbonate Improves Thyroid Function in Predialysis Chronic Kidney Disease. Am J Nephrol 2010; 32: 549-556

[6] Hahn A, Ströhle A, Wolters M. Ernährung: Physiologische Grundlagen, Prävention, Therapie. 1. Aufl. Stuttgart: Wissenschaftliche Verlagsgesellschaft; 2005

[7] de Brito-Ashurst I, Varagunam M, Raftery MJ et al. Bicarbonate supplementation slows progression of CKD and improves nutritional status. J Am Soc Nephrol 2009; 20: 2075-2084

[8] Mahajan A, Simoni J, Sheather SJ et al. Daily oral sodium bicarbonate preserves glomerular filtration rate by slowing its decline in early hypertensive nephropathy. Kidney Int 2010; 78: 303-309

[9] Bellasi A, di Micco L, Santoro D et al. Correction of metabolic acidosis improves insulin resistance in chronic kidney disease. BMC Nephrol 2016; 17: 158-167

[10] Kopp KF, Schätzle-Schuler G, Pankiewicz $T$ et al. Korrektur der interdialytischen Azidose bei chronischen Dialysepatienten. Nieren- und Hochdruckkrankheiten 1987; 14: $145-147$

[11] Seyffart G, Böhne M, Ensminger $A$ et al. Natriumhydrogencarbonat per os bei Hämodialysepatienten. Ergebnis einer Studie. Krankenhaus Arzt 1987; 60: 177-183

[12] Park S, Kang E, Park S et al. Metabolic Acidosis and Long-Term Clinical Out-comes in Kidney Transplant Recipients. J Am Soc Nephrol 2017; 28: 1886-1897

[13] Kalani LR, Shihab FS. Acidosis and Kidney Allograft Survival. J Am Soc Nephrol 2017; 28: 1672-1674 